\title{
Passive Acoustic Mapping of Extravasation Following Ultrasound-Enhanced Drug Delivery
}

\section{Catherine Paverd ${ }^{1,2}$, Erasmia Lyka ${ }^{1,2}$, Delphine Elbes ${ }^{1}$, Constantin Coussios ${ }^{1}$}

1. Institute of Biomedical Engineering, University of Oxford, United Kingdom

2. Joint first author

E-mail: catherine.paverd@eng.ox.ac.uk

August 2018

\begin{abstract}
.
The amount and distribution of chemotherapeutic agents delivered to tumours can vary significantly due to tumour heterogeneity, even under Focussed Ultrasound (FUS) assisted drug delivery regimes. The ability to non-invasively localise cavitation nuclei of a similar size to therapeutic drugs, both within the vasculature and tumour tissue, may provide a useful marker of ultrasoundenhanced drug delivery and extravasation. Solid polymer based nanoscale cavitation nuclei, under FUS excitation, have previously been shown to extravasate into tissue-mimicking phantoms, and to increase drug delivery in murine tumour models in vivo. Here we show in a tissue-mimicking material that these nuclei, once extravasated under FUS excitation, are still acoustically active and can be non-invasively localised using Passive Acoustic Mapping (PAM). By using a high resolution dual linear array setup in conjunction with adaptive beamformers, we demonstrate that the average 'Maximum Distance' of a PAM pixel to an extravasated particle across experiments is $0.4 \pm 0.2 \mathrm{~mm}$. Although the primary objective of the paper is to show that extravascular cavitation can be used as evidence of successful drug extravasation in a tissue-mimicking phantom, we also recognise the physical and computational limitations of using a high resolution dual array setup with adaptive beamformers. Thus as a secondary objective, we evaluate tradeoffs between adaptive and non-adaptive beamformers, as well as between dual and single array geometries. When compared to a conventional beamformer, adaptive beamformers reduce the maximum distance of PAM pixels to extravasated particles from an average of $2.4 \pm 0.7 \mathrm{~mm}$ to $1.8 \pm 0.6 \mathrm{~mm}$ in the single array case. The distance is further reduced to $0.4 \pm 0.2 \mathrm{~mm}$ using the dual array configuration, thereby demonstrating that increasing the solid angle spanned by the PAM array aperture significantly improves drug delivery localisation. Future work will test the applicability of PAM-based monitoring of ultrasoundenhanced drug delivery in vivo.
\end{abstract}

Keywords: Focussed Ultrasound, Nanoscale Cavitation Nuclei, Passive Acoustic Mapping, Adaptive Beamforming 


\section{Introduction}

The use of Focussed Ultrasound (FUS) in conjunction with micro- and nanoscale cavitation nuclei, for both diagnostic and therapeutic applications, is rapidly increasing. Many of these applications rely primarily on the physical effects caused by inertially and non-inertially cavitating bubbles. The primary diagnostic application of cavitation nuclei is for improving the Signal to Noise ratio (SNR) in Contrast-Enhanced Ultrasound and Doppler Imaging (Feigenbaum et al., 1970) (Dijkmans et al., 2004). Examples of therapeutic applications of FUS that rely on cavitation effects, both with and without added cavitation nuclei, include ultrasonic heating of tissue for ablation of cancer tumours (Kennedy et al., 2004), sonothrombolysis for the dissolution of blood clots (Datta et al., 2006), enhanced drug delivery to tumours (Carlisle et al., 2013), opening of the blood-brain barrier for drug transport (Hynynen et al., 2005), and transdermal sonophoresis (Mitragotri et al., 1995).

Two key factors have enabled these applications. First, the introduction of cavitation nuclei at the target site has allowed for inertial and non-inertial cavitation to be caused at relatively low pressure amplitudes. This has been essential in ensuring targeted interventions with minimal off-target side-effects. Second, the development of real-time, non-invasive monitoring strategies has contributed to the assessment of safety and efficacy in many of the aforementioned applications, and hence increased their adoption.

The first deliberate use of cavitation nuclei in ultrasound occurred in the early 1970s (Feigenbaum et al., 1970). Since then, multiple classes of cavitation nuclei have been developed, including microbubbles, nanobubbles and nanodroplets. Whilst the use of these cavitation nuclei enable many treatments, there are two factors that limit their applicability. First, the large size of microbubbles relative to human vessel pore sizes confines these agents to act within blood vessels. This is true even in vessels with large pore sizes, such as those found in the leaky vasculature of cancer tumours, where pore sizes range from approximately 100 to $1200 \mathrm{~nm}$ (Netti et al., 2000) (Peer et al., 2007) (Uzgiris, 2008) (Hobbs et al., 1998) (Dellian et al., 2000). Second, while some types of microbubbles can be stable in circulation (for example, polymer-shelled microbubbles), others have limited stability (Sirsi and Borden, 2010) and are rapidly destroyed if inertial cavitation is initiated by FUS. In the case of nanodroplets, sustained cavitation of a medium containing perfluorohexane nanodroplets has been achieved at peak rarefactional pressures of $12.6 \mathrm{MPa}$ at $0.5 \mathrm{MHz}$, however it is possible that the sustained cavitation activity is due to the resorption of nanodroplets into liquid form, which in turn decreases the cavitation threshold of the medium and allows for sustained cavitation, rather than the nanodroplets acting as individual cavitation nuclei (Vlaisavljevich et al., 2016).

A recently introduced ultrasound responsive cup-shaped polymeric nanoparticle with trapped gas in its cavity (know as a 'nanocup') has been shown to 
successfully initiate and sustain exclusively inertial cavitation activity over prolonged periods of time when compared to commercially available micron-sized cavitation agents (Kwan et al., 2015a). Recent work has demonstrated that nanocups, under ultrasound exposure, facilitate drug extravasation through the leaky tumour vasculature, thereby providing improved penetration and distribution of the drug into tumour tissue when compared to delivery of the drug alone (Kwan et al., 2015b). Previous studies have also investigated the role of inertial and noninertial cavitation both in vitro and in vivo (Bazan-Peregrino et al., 2012)(BazanPeregrino et al., 2013). These studies demonstrate that sustained non-inertial cavitation achieves negligible levels of additional extravasation compared to passive delivery alone, whilst sustained inertial cavitation achieves significant levels of omnidirectional extravasation in tumours. Thus an inertial cavitation scheme, enabled by the characteristics of the nanocups, is used in the present work.

To visualise nanocup activity, a cavitation monitoring technique is required. Several such techniques exist, such as interleaved B-Mode monitoring (Vaezy et al., 2001) and Magnetic Resonance Imaging (MRI) (Hynynen et al., 2001), but these methods are either impractical or do not directly measure inertial cavitation in real time, and hence are not suitable for mapping extravasated nanoparticles. However in the last decade, a technique known as Passive Acoustic Mapping (PAM) has been introduced (Gyöngy et al., 2008). During PAM, acoustic signals from collapsing bubbles are passively recorded on a set of receivers. The received signals are then filtered and combined using one of several beamforming techniques in order to generate an estimate of the power of wideband sources in a region, and hence to generate maps of the spatial distribution of sources in that area.

Several beamforming approaches have been proposed in the context of PAM, both in the time domain (Gyöngy et al., 2008) (Coviello et al., 2014) and in the frequency domain (Salgaonkar et al., 2009) (Haworth et al., 2012) (Arvanitis et al., 2016) (Lu et al., 2018). Broadly, these techniques can be categorised into conventional (non-adaptive), and optimal (data-adaptive) beamformers. In conventional beamformers, an equal weighting is applied to every received signal during the beamforming process. However in adaptive beamformers, statistical information contained in the received signals is used to determine a variable weighting for each signal, with the goal of minimising the contribution from unwanted signals to the reconstruction. PAM has already been shown to provide successful monitoring of cavitation during High Intensity Focussed Ultrasound (HIFU) tissue ablation (Jensen et al., 2013), drug delivery studies (Choi et al., 2014), and blood-brain barrier disruption treatments (Arvanitis et al., 2012). The resolution of PAM varies based on the type of beamformer used, and is further dependent on the diffraction pattern of the detector (Gyöngy and Coussios, 2010)(Gray et al., 2018). Therefore setups with larger apertures provide better resolution, which is essential for mapping extravasated particles. However the 
geometric space requirements for larger apertures may not always be practical, and thus these setups can have limited clinical applicability.

In the present work the authors aim to demonstrate that nanoscale cavitation nuclei, once extravasated under ultrasound excitation, are still acoustically active and can be non-invasively located in a tissue-mimicking phantom. We also recognise that the best spatial resolution for this purpose is achieved at significant computational cost and physical complexity, and thus we further investigate the effect of less complicated physical setups and acoustic beamformers on the accuracy of post-extravasation particle mapping. A comparison of the accuracy and a discussion of the tradeoffs associated with each of the approaches is also presented, with the aim of determining the best beamformer for this application in a clinically relevant setup.

\section{Experiment Materials and Methods}

\subsection{Experimental Procedure}

A series of flow channel experiments were performed in a vertically oriented flow channel embedded in an agarose (agar) gel phantom, as shown in figure 1 . The experimental procedure was as follows. First, nanocups were pumped through the flow channel at a constant rate of $0.3 \mathrm{ml} / \mathrm{min}$ using a syringe pump (Aladdin Single Syringe Pump, AL-1000, World Precision Instruments, Hertfordshire, UK). Initial ultrasound excitations were then performed using a dual FUS transducer configuration with the aim of causing extravasation of the nanocups from the flow channel into the surrounding phantom material. The flow channel was then flushed for 30 minutes with ultrapure, degassed water. The flow of water was halted and a second sonication was performed at each target location, with the aim of reexciting nanocups that had been embedded in the phantom material during the initial sonication. During both the initial and second sonications, acoustic emissions were recorded on two L11- 4 linear arrays using a Verasonics Vantage 256 channel research ultrasound system. Once the sonications were complete, the phantom was removed and cut transversely and in cross section in order to verify the location of extravasated particles using fluorescence microscopy with a Nikon TI Eclipse microscope.

From figure 1 it can be seen that the axial-transverse planes of both ultrasound transducers are perpendicular to the long axis of the flow channel. When cut transversely, a significant part of the long axis of the channel is visible on microscopy (with the edges of the channel appearing as two parallel lines), and the incident direction of the ultrasound is out of plane relative to the microscopy slide. When cut in cross section, the full circular border of the channel is visible, and the incident direction of the ultrasound is in plane with the microscopy slide. 
SIDE VIEW
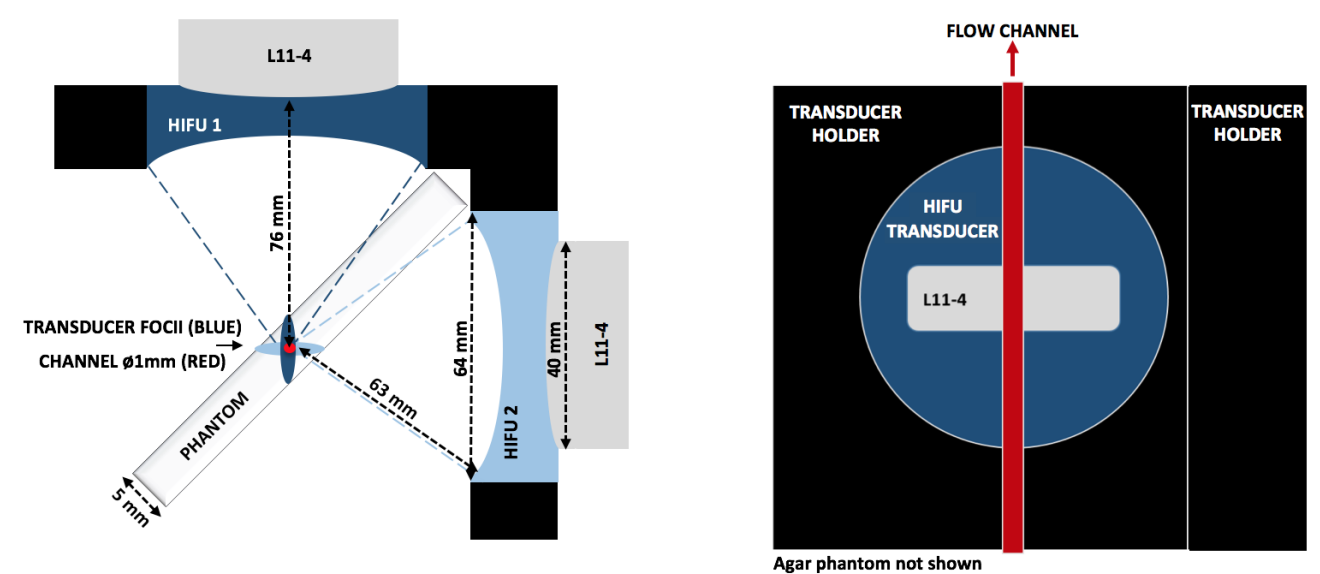

Figure 1. Left: Top view of the experiment setup showing two confocally aligned FUS transducers, with two L11-4 linear arrays coaxially aligned with each transducer. Right: Side view of the experiment setup showing the flow channel (red) at the focus of the FUS transducer and L11-4 array.

\subsection{Phantom Design}

Ultrapure Low Melting Point agar gel (ThermoFisher Scientific Inc., Waltham, MA) was selected for the tissue mimicking phantom as it ensured a pore size on the order of several hundred nanometres, which is similar to that found in tumour vessels (Narayanan et al., 2006). To prepare the phantom, a $1 \%$ by weight solution of agar powder was mixed into filtered, de-ionised water and degassed for a minimum of 30 minutes, heated to $70^{\circ} \mathrm{C}$, poured into a phantom mould containing a $1 \mathrm{~mm}$ diameter stainless steel rod in the channel position, and then left overnight to set in a $4^{\circ} \mathrm{C}$ fridge. Once set, the steel rod was removed, leaving an agar bounded flow channel.

\subsection{Cavitation Nuclei}

The cavitation nuclei used in these experiments are polystyrene based, cup shaped particles with a mean diameter of $420 \mathrm{~nm}$, as shown by the Dynamic Light Scattering measurement in figure 2. These particles were formed by a seeded polymerisation technique and air-dried to entrap a gas nanobubble in the cavity, as described by Kwan et al. (2015b). In addition, the cups were fluorescently labelled with Nile Red dye (Sigma-Aldrich Inc., Merck KGaA, Darmstadt, Germany) during fabrication. Under ultrasound excitation, these nanocups exclusively exhibit inertial cavitation above a certain pressure threshold, and this cavitation threshold increases as the size of the entrapped gas bubble decreases. The entrapped gas bubble is assumed to have the same diameter as the cavity diameter (or inner diameter) of a nanocup. Kwan et al. demonstrate that for nanocup cavity diameters of $100 \mathrm{~nm}$, the 


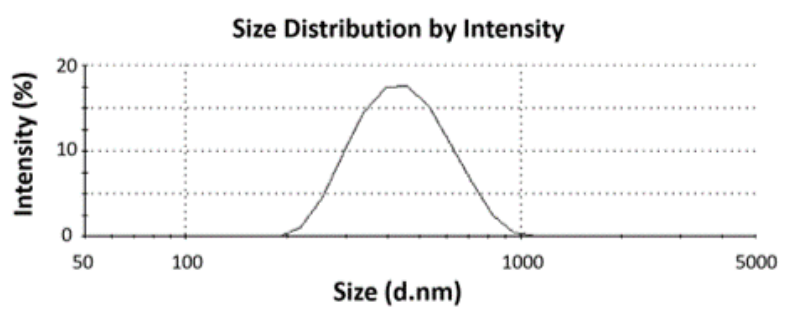

Figure 2. Dynamic Light Scattering measurement of nanocup size (Zetasizer Nano, Malvern Panalytical, Worcestershire, UK) indicating mean size of 420nm, with a Polydispersity Index of 0.08 .

inertial cavitation threshold is approximately $2 \mathrm{MPa}$ at $0.5 \mathrm{MHz}$, and decreases below $1 \mathrm{MPa}$ peak rarefactional pressure for cavity diameters greater than $300 \mathrm{~nm}$ (Kwan et al., 2015a). In this set of experiments, the smallest measured particle size was approximately $200 \mathrm{~nm}$ (as shown in figure 2). Thus the minimum cavity diameter is assumed to be between 100 and $200 \mathrm{~nm}$. This minimum size range was used to determine the selection of acoustic pressures described in section 2.5.

\subsection{Acoustic Setup}

FUS: Two $0.5 \mathrm{MHz}$ confocal FUS transducers (H107, outer diameter $64 \mathrm{~mm}$, focal length $63.2 \mathrm{~mm}$, Sonic Concepts, Bothell, WA) with full width half maximum (FWHM) focal width of $3 \mathrm{~mm}$ and FWHM focal depth of $21 \mathrm{~mm}$ were arranged as shown in figure 1 . The dual transducer setup was used to increase spatial targeting, resulting in a FWHM focal area of $1 \times 6 \mathrm{~mm}$, with reduced side lobes located approximately $1 \mathrm{~mm}$ away on either side of the focus, as shown in Supplementary Material, Part B. This dual transducer setup was further intended to reduce the effect of radiation force in a single direction when compared to a single transducer producing the same focal pressure. Thus this configuration increases the relative contribution of cavitation activity to particle extravasation and, by extension, the probability of extravasation occurring in any direction around the channel.

PAM: Monitoring of cavitation activity was achieved by PAM using signals recorded on two L11-4 linear arrays (centre frequency $6.25 \mathrm{MHz}$, bandwidth 4 $11 \mathrm{MHz}, 128$ elements, pitch 0.298 - $0.302 \mathrm{~mm}$, Verasonics Inc., Redmond, WA), each aligned coaxially with one of the FUS transducers. This perpendicular arrangement increased the effective array aperture, and enabled the best possible resolution (ordinarily only in the transverse direction) in both the transverse and axial directions. The linear arrays were connected to a Verasonics Vantage ultrasound research system (Verasonics Inc., Redmond, WA) on which signals received by each element were recorded for use in offline PAM processing. When PAM reconstruction was completed with data from both linear arrays, it is referred to as a Dual Array case in this paper. If signals from one of the two arrays were used in PAM 
reconstruction, it is referred to as a Single Array case.

\subsection{Experiment Parameters}

Two different types of acoustic exposure regimes were used in this experiment. In this article all pressures are reported as Peak Rarefactional Pressure (PRP) and pressures were measured using a $0.4 \mathrm{~mm}$ needle hydrophone calibrated at $0.5 \mathrm{MHz}$ by the manufacturer (HNA Needle Hydrophone, Onda Corp., Sunnyvale, CA). For the initial sonication, where the goal was to cause extravasation, a $5 \%$ duty cycle at a constant pressure of $1.6 \mathrm{MPa}$ and $\mathrm{PRF}$ of $3 \mathrm{~Hz}$ was used. The $5 \%$ duty cycle ensured that extravasation was readily achieved, whilst minimising thermal effects in the phantom, and the $3 \mathrm{~Hz}$ PRF was selected to ensure sufficient time for data to be saved on the Verasonics. A pressure of 1.6 MPa was selected as it is the minimum pressure at which the probability of cavitation is $100 \%$ for the nanocups used. A flow rate of $0.3 \mathrm{ml} / \mathrm{min}$ was used to ensure that the nanocup solution was replaced at the focus between each pulse.

In contrast, the second sonication (post-clearance) was designed to facilitate inertial cavitation of extravasated particles of all sizes, whilst minimising any further movement of these particles into the phantom. As smaller nanocups have slightly higher inertial cavitation thresholds, a pressure ramp was used on the second sonication in order to map as many extravasated nanocups as possible. The minimum PRP was the same as the pressure for the first sonication, 1.6 MPa. This pressure was then gradually increased to $2.1 \mathrm{MPa}$ over the course of the second sonication. The maximum PRP of $2.1 \mathrm{MPa}$ was selected as it is above the PRP cavitation threshold for the smallest size of nanocups used in this experiment, but is still below the PRP cavitation threshold of the phantom material (as evidenced by a lack of cavitation activity during controls at the same pressure). In addition, at the maximum PRP used, the PRP in parts of the sidelobes was also significant enough to exceed the PRP cavitation threshold for some of the larger nanocups, thereby allowing for post-extravasation mapping of nanocups in a larger region. To minimise further transport of the cavitation nuclei into the phantom, and to allow for sufficient time between pulses for the computation and display of real-time PAM images, the duty cycle and PRF were reduced to $0.05 \%$ and $1 \mathrm{~Hz}$ respectively.

\section{Image Processing Methods}

\subsection{Image Acquisition}

Upon completion of the ultrasound exposures, the agar phantom was removed and imaged in transverse and cross section using a Nikon TI Eclipse microscope (Nikon Corp., Tokyo, Japan) with tetrarhodamine (TRITC) filter under 4x magnification, to verify the location of extravasated cavitation nuclei. Areas of fluorescence within 
the agar at the ultrasound target locations indicated the presence of extravasated nanocups.

\subsection{Image Processing Procedure}

After acquisition, offline processing of the PAM signal data and microscopy images was completed using Matlab (MathWorks Inc., Natick, MA) and ImageJ (National Institute of Health, Rockville, MD) as described below.

Microscopy: The microscopy images from a cross-sectional segment of agar gel at the ultrasound focus were compressed into a single image using a Maximum Intensity Projection. This image was then loaded into Matlab and a set angle rotation of $135^{\circ} \pm 5^{\circ}$ was applied. This rotation was required because during the experiment, for ease and accuracy of microscopy, the cross sectional segments of the agar phantom were imaged at an angle that was different to their original setup orientation (such that a straight edge of the section of agar being imaged could be aligned with an axis of the microscopy movement stage). The PAM co-ordinate system was chosen as the reference co-ordinate system, and therefore the microscopy rotation was required in order to orient the microscopy image correctly. Although the microscopy images were obtained in such a manner as to minimise alignment error, we could not ensure that we had performed the alignment perfectly, or that the phantom was at exactly 45 degrees to the linear arrays. Thus we allowed for the rotation to vary by a small amount $\left( \pm 5^{\circ}\right)$ for each experiment. Once rotated, an automated circle detection algorithm in Matlab was used to identify the channel wall in the image. An iterative automated thresholding procedure using the IsoData method (Ridler, 1978) was applied in order to automatically identify a microscopy Region of Interest (RoI).

$P A M$ : The location of the channel relative to each linear array was identified using B-Mode data collected while the channel was filled with air, before the solution of nanocups was introduced. To ensure that the start of the flow did not disturb the channel, the identified location was confirmed using single array PAM maps from each linear array acquired during the initial sonication. Once the location of the channel relative to each probe was determined, the coordinates of the elements in one of the linear arrays were referenced to the other array, thereby creating a larger aperture array for PAM reconstruction in the dual array case. PAM reconstruction for each probe individually and for both probes together was performed using the three different beamformers, as described in section 3.3. For the second sonication, PAM maps displaying activity above baseline noise were identified and thresholded using the thresholds described in section 3.4. The resulting frames were added to form a composite PAM image, thereby encapsulating data from the entire exposure period. The composite PAM map was then upscaled to the same size as the microscopy image using bicubic interpolation and thresholded to remove values outside of the original PAM map range. The channel location previously identified 
was added to the composite image, allowing for an uncertainty of one B-Mode pixel $(0.12 \mathrm{~mm})$, which is the equivalent distance of half a PAM pixel.

Merging and Analysis: The composite PAM map and the binary microscopy image were then co-registered using the channel location, and the two images were fused to create the resulting images shown in figure 4 and 5. In addition, metrics that aim to quantify performance in relation to energy and pixel distance were calculated.

\subsection{PAM Processing}

The acoustic emissions recorded during the second sonication were filtered using a 4 $10 \mathrm{MHz}$ bandpass filter to isolate the broadband emissions due to inertial cavitation within the bandwidth of the arrays. PAM maps of the sources of the filtered acoustic emissions were generated for both the single and dual array configurations using three different beamforming approaches: Time Exposure Acoustics (TEA) (Gyöngy et al., 2008), Robust Capon Beamforming (RCB) (Coviello et al., 2014), and Robust Linear Programming Beamforming (RLPB) (Lyka et al., 2018). TEA is a representative non-adaptive beamformer. It recreates the energy in a pixel of interest by time delaying the signals received on each array element by an appropriate amount before summing the signals using equal weights, thereby determining the total signal energy in a particular pixel. Other non-adaptive beamformers employ a similar strategy either in the time or frequency domain, and include Passive Cavitation Imaging (PCI) (Haworth et al., 2012) and Angular Spectrum PAM (AS-PAM) (Arvanitis et al., 2016). Such non-adaptive approaches have very low computational cost, but result in low spatial resolution as the statistical characteristics of the recorded signals are not taken into consideration. In contrast to conventional beamformers, both RCB and RLPB are data-adaptive beamformers, since the calculation of the array element weights is the solution of an optimisation problem based on information from the recorded signals. Adaptive approaches provide improved resolution through better interference suppression, as well as by the mitigation of minor array calibration errors. While RCB assumes the signals follow a Gaussian distribution and attempts to minimise the variance of the array output, RLPB makes no prior assumptions on the signal distribution, and factors in the Higher Order Statistics of the signals by attempting to minimise the $\ell_{\infty}$-norm of the array output (Jiang et al., 2014). Other data-adaptive beamformers include an Eigenspace-based implementation of RCB (Lu et al., 2018).

\subsection{PAM Threshold Optimisation}

Accurate detection and localisation of regions of acoustic sources within a PAM map requires the application of a relevant threshold. To determine an optimal threshold, a series of numerical simulations were performed on 2D bubble clouds 
of various spatial distribution, bubble population and radius. In the simulations, when the bubble distribution was varied, the number of bubbles was held constant and conversely, when the number of bubbles was varied, the distribution was held constant. A full table of simulation parameters can be seen in the Supplementary Material, Part C.

Both the single and dual array configurations were investigated, with the arrays situated $75 \mathrm{~mm}$ away from the bubble cloud, as in the in vitro experimental setup. Bubble oscillations due to an incident acoustic field identical to that used in the in vitro setup were modelled using the Keller-Miksis model under the assumption of an infinite medium of incompressible fluid (Keller and Miksis, 1980) (Collin and Coussios, 2011). Pressure waves radiated from the excited bubbles were spherically propagated towards either one or two linear arrays, whose bandwidth and configuration matched that of a Verasonics L11-4 probe, and white Gaussian noise was added to the array to mimic noise due to electronics. The resulting acoustic signals were beamformed using RCB, and different thresholds were applied, which range between $-20 \mathrm{~dB}$ and $0 \mathrm{~dB}$ with respect to the maximum PAM energy in the map. The application of a threshold yielded an RoI for the PAM map. Then, for each simulation, two measures of localisation were calculated: the percentage of bubbles located inside the RoI, and the percentage of the RoI covered by bubbles. The optimal threshold is defined as the best compromise between capturing all of the bubbles in a given area, and accurately representing only areas in which bubbles are present in a map. This optimal threshold is therefore represented by the intersection of the aforementioned two metrics, as shown in figure 3. The parameters tested in these numerical simulations result in a mean optimal threshold of $-2.0 \pm 0.58 \mathrm{~dB}$ for the single array configuration, and $-5.3 \pm 0.87 \mathrm{~dB}$ for the dual array configuration. These thresholds were used for the PAM reconstructions discussed throughout the remainder of this paper.

\section{Results}

\subsection{Qualitative Results}

Ten experiments were conducted with the procedure described in section 2.1. Three representative fused images created using the dual array configuration and the RLPB beamformer are shown in figure 4, and the full set of resulting images can be seen in the Supplementary Material. In the images, green represents the microscopy RoI (indicating the presence of extravasated fluorescent nanocups), magenta indicates the thresholded composite PAM image, white indicates areas of overlap between PAM and the microscopy RoI, the red circle shows the location of the channel, the red arrows indicate the direction of ultrasound, and the white arrows indicate the axial direction of view of the linear arrays used during the PAM reconstruction.

Thus in the images presented, regions of white indicate 'on-target' PAM energy 

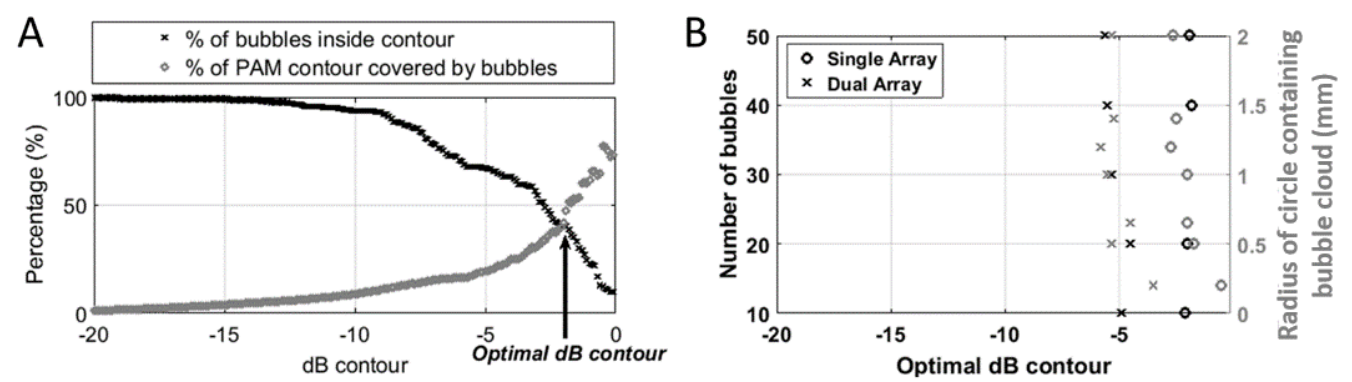

Figure 3. A) Example of the calculation of the optimal dB contour for PAM thresholding. The optimal contour is taken as the intersection of two metrics. The first represents the percentage of bubbles inside each $\mathrm{dB}$ contour, and the second represents the percentage area of the $\mathrm{dB}$ contour covered by bubbles. B) The optimal dB threshold for single and dual arrays for a range of bubble populations and distributions. The left Y-Axis represents the number of bubbles and the right Y-Axis represents the radius of the circle containing the bubbles in the imaging plane of the linear arrays.
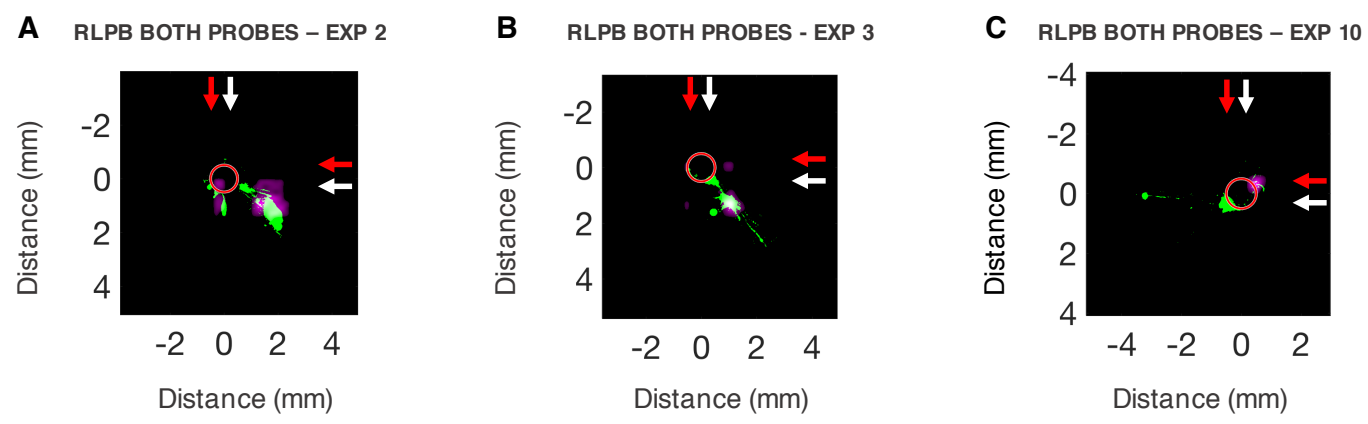

Figure 4. Three representative fused images created using the dual array configuration and the RLPB beamformer. Green represents the microscopy RoI (indicating the presence of extravasated fluorescent nanocups), magenta indicates the thresholded composite PAM image, white indicates areas of overlap between PAM and the microscopy RoI, the red circle shows the location of the channel, the red arrows indicate the direction of ultrasound, and the white arrows indicate the axial direction of view of the linear arrays used during the PAM reconstruction.

and regions of purple indicate 'off-target' PAM energy. Hence a high ratio of on-target to off-target PAM energy is indicative of good localisation performance, provided the off-target energy is still clustered in the general area of extravasated particles. Experiment 2 in figure $4 \mathrm{~A}$ demonstrates average localisation performance, in which there is a relatively good ratio of on-target to off-target energy, and the off-target energy is still located in the immediate vicinity of extravasated particles. Experiment 3 (figure 4B) demonstrates a high percentage of on-target energy, but also shows a small amount of off-target energy located slightly further away from the extravasated particles than in the previous case. Finally, Experiment 10 (figure 4C) 


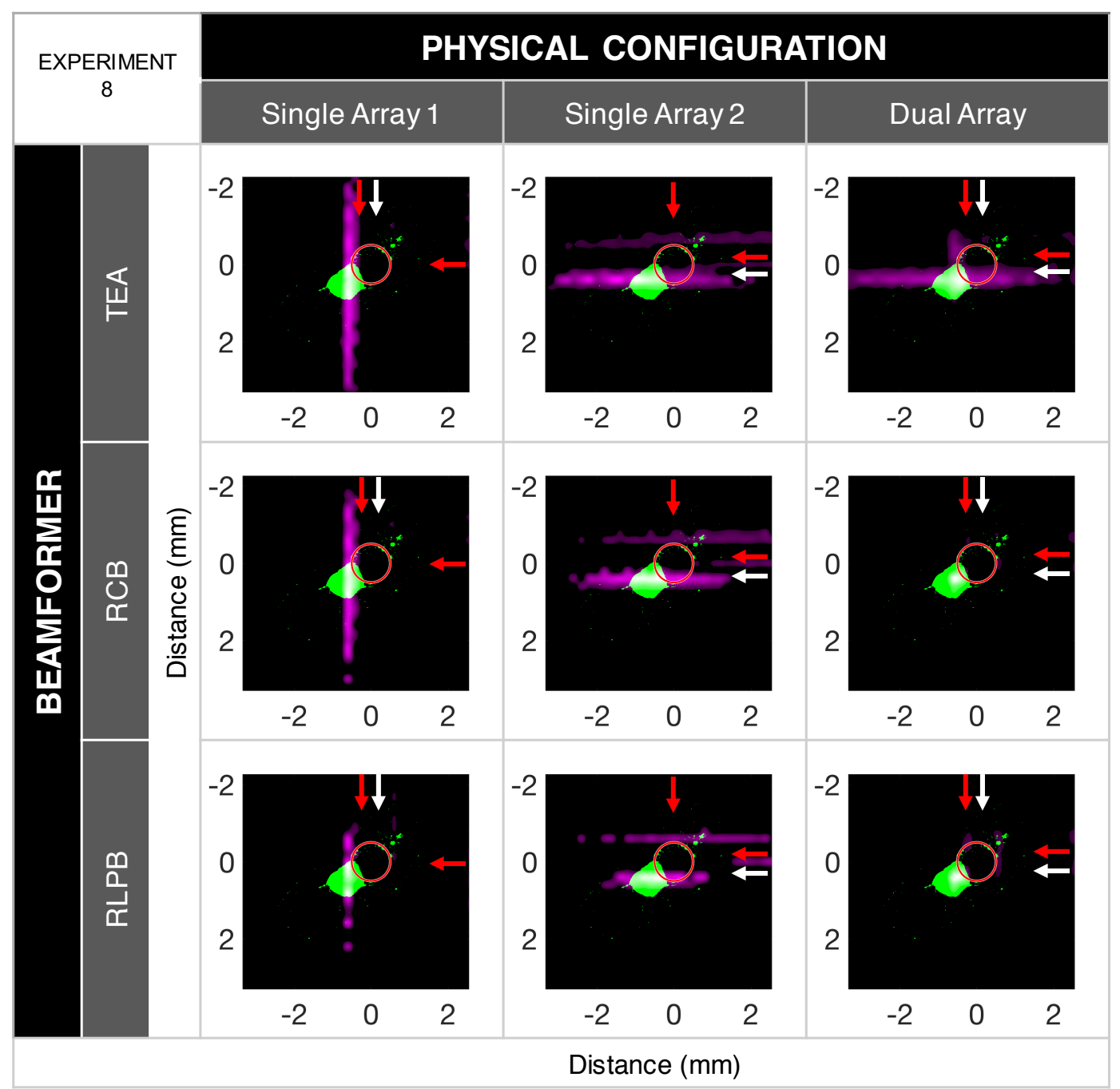

Figure 5. The fused images for both the single and dual array configurations for each of the three beamformers evaluated. Green represents the microscopy RoI (indicating the presence of extravasated fluorescent nanocups), magenta indicates the thresholded composite PAM image (generated from individual PAM frames collected during the second sonication), white indicates areas of overlap between PAM and the microscopy RoI, the red circle shows the location of the channel, the red arrows indicate the direction of ultrasound, and the white arrows indicate the axial direction of view of the linear arrays used during the PAM reconstruction.

shows very little on-target energy, even though the signal is located in the correct region. In addition, in Experiment 10, there is a significant 'green-only' area, indicating that even though particles have extravasated to that area, they have not been re-excited during the second sonication. The reasons for this are discussed in section 5 .

Similar to the examples shown in figure 4, in all of the other experiments performed, several PAM frames showing cavitation events which overlapped with extravasated particles were always recorded during the second sonication. On the 


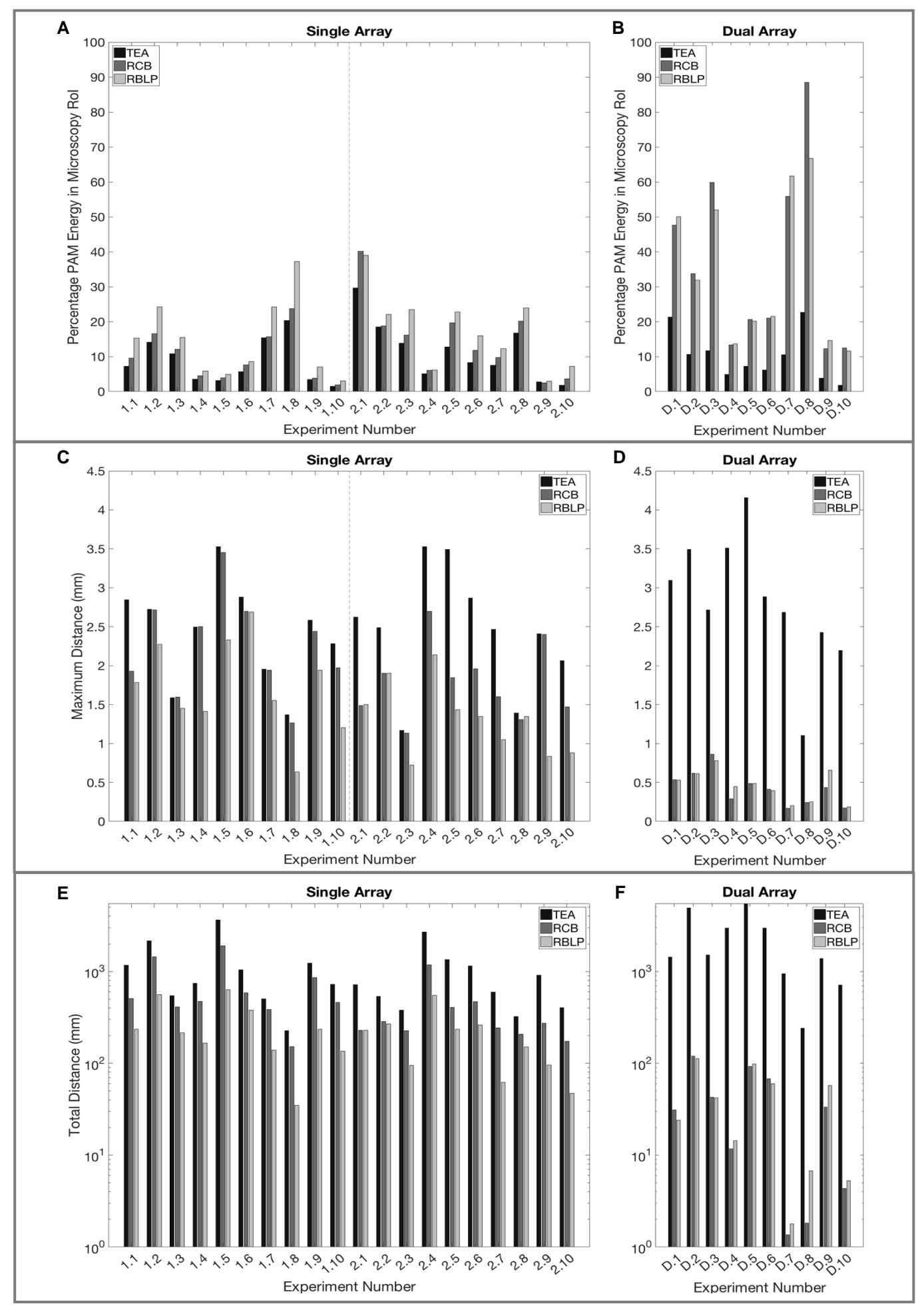

Figure 6. A \& B) PAM energy within the microscopy RoI as a percentage of total PAM energy for all beamformers for (A) the single or (B) dual array case. C \& D) The maximum euclidean distance of a PAM pixel to the nearest pixel inside the microscopy RoI, for (C) the single and (D) dual array case. E \& F) The sum of the distance of every PAM pixel outside of the microscopy RoI to the nearest pixel inside the microscopy RoI. This metric represents the 'clustering' of PAM around extravasated particles for (E) the single and (F) dual array case. 
second sonication, cavitation events were recorded at the minimum PRP of 1.6 MPa (the same PRP as used in the initial sonication), and also at greater pressures (up to the maximum PRP of 2.1 MPa). No such signal was recorded and no extravasation was evident in control experiments employing an identical experimental protocol and acoustic exposure parameters, but using fluorescent spheres of $300 \mathrm{~nm}$ in diameter instead of nanocups. This result indicates that nanocups, initially extravasated under ultrasound excitation, can be acoustically re-excited and localised using PAM.

To assess clinically relevant setups, the single array case was also evaluated. A representative qualitative result that illustrates both single and dual array cases is given in figure 5. A degradation in performance is shown by a larger region of PAM energy located further away from the extravasated particles, and is clearly evident in the axial direction of the single array case compared to the dual array case. In addition, the conventional TEA beamformer demonstrates greater spreading of the PAM energy over a region in comparison to the adaptive beamformers. Thus the single array cases using TEA demonstrate the worst localisation performance.

\subsection{Quantitative Results}

To quantify the performance change, three metrics are presented in figure 6 . The first metric, 'Percentage Energy', measures the percentage of PAM energy inside the microscopy RoI for all configurations as a percentage of total PAM energy (i.e. the white area divided by the total of the magenta and white areas). This metric therefore indicates how much of the total PAM energy originates from a region of extravasated particles. Therefore, in this graph, a higher percentage of energy is generally considered to indicate better performance. However, whilst a high percentage of energy may overlap with extravasated particles, it is also important to consider where the remainder of the energy is distributed. Thus we introduce a second metric, 'Maximum Distance', which displays the maximum distance of any PAM pixel to the nearest extravasated particle for the different setups and beamformers. Here, a greater distance is normally indicative of degraded performance. This is especially evident in the single array case where PAM energy is significantly elongated in the axial direction. Finally, a third 'Clustering' metric is used to evaluate both the number and distance of off-target pixels in order to ascertain how tightly clustered the PAM energy is around a region of extravasation. This metric is calculated as the summation of the distance of every PAM pixel that is outside of the microscopy RoI to the nearest pixel within the microscopy RoI. Thus a high value implies that the PAM energy is not clustered near to a region of extravsated particles. However this metric may still be biased by a significant number of 'off-target' PAM pixels, or the distant location of a small number of PAM pixels. Thus it is useful to examine information across all three metrics simultaneously when analysing performance.

For example, comparing Experiments 2 and 10 (shown in figure 4) according to 
the Energy metric, Experiment 2 has approximately three times the percentage of 'on-target' pixels than Experiment 10. However, from the Maximum Distance and Clustering metrics it can be seen that Experiment 2 has more PAM pixels located further away from extravasated particles, and hence Experiment 2 has a greater spread of energy around the extravasated particles. If the results are normalised to a single beamformer within each experiment and compared, statistically significant differences exist between every beamformer for the single array case, and between TEA and RCB, and TEA and RLPB for the dual array case (1-way ANOVA, $\mathrm{p}<0.05)$. No statistically significant difference exists between RCB and RLPB for the dual array case for any metric. Overall the adaptive beamformers, when compared to TEA, reduce the maximum distance of PAM pixels to extravasated particles from an average of $2.4 \pm 0.7 \mathrm{~mm}$ to $1.8 \pm 0.6 \mathrm{~mm}$ in the single array case. The distance is further reduced to $0.4 \pm 0.2 \mathrm{~mm}$ using the dual array configuration. In terms of PAM energy co-localised with extravasated particles, this increases from an average of $10.1 \pm 7.5 \%$ to $14.2 \pm 10.1 \%$ with adaptive beamformers in the single array case, and further increases to $35.5 \pm 23.0 \%$ when using the dual array system geometry. Finally, on the Clustering metric, the mean total distance across experiments for TEA in the single array case is $1051 \pm 861 \mathrm{~mm}$, which reduces to $391 \pm 379 \mathrm{~mm}$ with adaptive beamformers in the single array case, and further decreases to $41 \pm 39 \mathrm{~mm}$ with dual array adaptive beamformers.

\section{Discussion}

From the qualitative results it is clear that nanocups can be re-excited, and the authors believe that this re-excitation is possible for two reasons. First, during the initial sonication, it is likely that not all nanocups would have cavitated, but rather that some would have been forced into the agar gel as a result of the mechanical effects of neighbouring cavitating nanocups. These nanocups would then still contain their entrapped gas pocket, and hence would retain the ability to cavitate under ultrasound excitation. Second, it is possible that gas from collapsed bubbles reforms on the surface of the solid polymer cup base, and hence enables the nanocup to cavitate again during the second sonication. This is possible because the polymeric base of the nanocups is hydrophobic, and therefore forms an energetically attractive structure for the re-formation of a gas bubble. In reality it is likely to be a mixture of these two hypotheses, and further work is required to completely elucidate the underlying mechanisms.

In section 2.4, it is noted that the dual array FUS transducer configuration increases the relative contribution of cavitation activity to particle extravasation and, by extension, the probability of extravasation occurring in any direction around the channel. Although the effect of radiation force cannot be completely eliminated by this setup, previous work has demonstrated that an acoustic radiation force in 
the absence of cavitation nuclei does not have a significant effect on the transport of particles (on the order of hundreds of nanometers in size) when compared to cavitation-mediated delivery (Lea-Banks et al., 2016). Furthermore, during the controls performed for this experiment (in which fluorescent polymeric spheres of similar size and density to the nanocups were used), the lack of significant extravasation projections indicates minimal transport due to acoustic radiation force. However, it is important to note that the acoustic radiation force on bubbles once nucleated from the nanocups may have a significant impact.

It was also noted in the qualitative results section that in some cases, the PAM signal is not located in all regions in which extravasation is observed (which is indicated by small region of white compared to the total green region). This may be due to several factors. First, relatively long extravasation projections may be formed in any direction around the channel during the initial sonication due to the unpredictable interactions between cavitation forces, acoustic radiation forces, microstreaming effects and the local structural properties of the agar gel. However, the focal region of the FUS setup is extremely small and highly targeted to the channel, so only the nanocups located in the focal region (or side lobes at high enough pressures) will cavitate. Therefore it is unlikely that extravasated nanocups in all areas of the phantom will be detected without steering the ultrasound focal area to cover the entire region. Second, although nanocups may have extravasated into a region that is still covered by the FUS focus, those extravasated nanocups may have lost their entrapped gas bubble during the initial sonication, and hence may have lost their ability to cavitate again during the second sonication, or the concentration of cavitating nanocups may be too low to produce detectable signals. Finally, diffusion and convection of nanocups following a cavitation event may occur on length-scales that are large relative to the region of the cavitation event alone. It should be noted however, that the focus of the current work was not to localise all extravasated particles, but rather to show that PAM signals received on the second sonication do originate from extravasated nuclei. For a more complete mapping of all extravasated particles, it would be necessary to steer the FUS focus or move the phantom relative to the transducers in order to ensure that all regions of the phantom are exposed to sufficiently high pressure on the second sonication. In addition, higher concentrations of cavitation nuclei may be required to ensure sufficient cavitation signal can be recorded for all regions of extravasation on the second sonication.

Finally, variability within each metric, and especially within the PAM Energy metric, may also be due to several factors. First, in cases where there is a high level of cavitation, the PAM energy is spread out extensively due to the Point Spread Function (PSF) of the system. Therefore when a large cavitation event is detected, the PAM map will show the peak energy at the source of the cavitation event, but will also show energy 'smeared' around that location. However the PSF of PAM is significantly larger than that of microscopy for both the single and dual 
array cases. Thus the fluorescence microscopy image of the groups of sub-micron cavitation nuclei that cause these cavitation events will not be as 'smeared' and will thus indicate a smaller (and more accurate) representation of the true location of extravasated nuclei. This is the primary reason why a significant level of PAM energy does not coincide with extravasated nanocups, as shown in the representative images in figures 4 and 5 . It is further clarified by noting the extremely low values in the Clustering and Maximum Distance metrics in figure 6 for the adaptive beamformers in the dual array case. The following subsection discusses variations between beamformers and array configurations in more detail. In addition, a low percentage energy may also be due in part to the loss of low fluorescence intensity areas of the microscopy images as a results of automated thresholding.

\subsection{Beamformer and Array Configuration}

For all beamformers, the choice of array configuration, and hence array aperture, has a direct impact on the spatial resolution of PAM, with a larger array aperture resulting in better resolution. In addition, the orthogonality of the arrays in the dual array case ensures that the resolution in both the transverse and axial directions is at least equivalent to the resolution in the transverse direction for a single array.

As can be seen in figure 5, adaptive beamformers have significantly smaller PSF and result in less spreading of the PAM energy. This performance improvement is due to the ability of adaptive beamformers to adjust signal weighting based on statistical characteristics of the received signals, as described by Coviello et al. (2014). Furthermore, in the case of the single array configuration, RLPB exhibited significantly better performance than RCB in terms of source localisation. Such behaviour illustrates the importance of considering not only the variance, but also the higher order statistics of the signals during beamforming for improved interference suppression, and ultimately, better source localisation. However in the dual array case, there is no significant difference between RCB and RLPB. This similarity in performance of the adaptive beamformers is most likely because the use of two arrays already significantly improves the PSF of the setup, and hence the reduction in PSF due to RLPB is less apparent.

In addition to the type of beamformer used, the physical array geometry has a significant impact on the system performance. The resolution of all of the beamformers used is dependent on array aperture. Therefore, by increasing the array aperture the PAM resolution, and hence the particle localisation performance, is significantly improved.

\subsection{Tradeoffs And Limitations}

Although the dual array setup with an adaptive beamformer provides the best performance for mapping extravasated particles, the use of two orthogonal arrays 
presents practical geometric challenges, and hence may only be applicable in very limited clinical settings.

Thus it is important to examine the results of the single array case for clinical relevance. In this case, RLPB significantly outperforms TEA and RCB, and is therefore a promising candidate for adoption into a clinically relevant setup.

However the use of adaptive beamformers compared to conventional beamformers increases computational cost. Of the adaptive beamformers, RLPB requires significantly more computational time than $\mathrm{RCB}$, as the beamformer is required to find the solution to a linear programming problem - an inherently iterative process that cannot be parallelised. In contrast, the most computationally expensive part of RCB is the eigenvalue decomposition of a covariance matrix. By comparison, for a typical map of 100 voxels and signals recorded for $120 \mu \mathrm{s}$ at $25 \mathrm{MHz}$, an RCB map is generated approximately 200 times faster than the corresponding RLPB map. Thus using a 2.6 MHz Intel Xeon CPU (E5-2650v2, Intel Corp., Santa Clara, CA), the achievable RCB PAM frame rate is $0.86 \mathrm{~Hz}$, while the corresponding RLPB frame rate would currently be $0.004 \mathrm{~Hz}$.

These timings are based on implementation of both algorithms in MATLAB using the available built-in functions. Therefore whilst the current implementation of RLPB is not fast enough for real-time applications, it is likely that by using optimised commercially available software for solving linear programming problems, in conjunction with data reduction strategies that do not significantly compromise signal quality, RLPB can be performed on clinically relevant timescales.

Even if the processing time can be reduced, the resolution of PAM created with a single array, particularly in the axial direction relative to the array, would not suffice in a clinical setting where sub-millimetre length scales are common. Thus further work is necessary to improve the resolution of these techniques for the single array case. Strategies to achieve this include moving the array closer to the target where possible, increasing the aperture in a clinically feasible manner (for example, by adding a second array at an obtuse angle, rather than at $90^{\circ}$ ), employing diffraction and attenuation correction (Gray et al., 2018), and utilising advanced imaging techniques such as superresolution (O'Reilly and Hynynen, 2013), in conjunction with the beamforming techniques mentioned here.

\subsection{Application to Drug Delivery}

One of the main applications of this work will be in enhanced drug delivery, where the ability to non-invasively localise cavitation nuclei of a similar size to therapeutic drugs, both within the vasculature and tumour tissue, may provide a useful marker of drug delivery. In particular, the dual transducer setup may be most useful in small animal studies and in in vitro studies where high spatial resolution is required. In addition, the dual transducer setup may also be used in some clinical applications where a dual acoustic window is available, such as in breast and head-and-neck 
oncological cases. Furthermore, the single array case with the most advanced beamformer may be used in clinical situations where length-scales are slightly larger and high spatial resolution is not as critical.

In typical drug delivery applications, a cavitation agent is used in combination with a drug. However, in this work we do not use an additional drug in the system, but rather use the nanocups themselves as model drug. This is because the nanocups have been designed to have a comparable overall size $(300-500 \mathrm{~nm})$ to many of the larger therapeutic agents for which they are meant to aid extravasation, including oncolytic viruses $(100-300 \mathrm{~nm})$, liposomes and polymerosomes $(100-250 \mathrm{~nm})$ and, to a lesser extent, therapeutic antibodies $(10-30 \mathrm{~nm})$. Only a subset of the nanocups are activated within any one acoustic pulse, and thus the remaining fluorescently labelled particles act as an adequate free drug model, as comparable transport length scales for nanocups and model or real co-administered drugs have been previously demonstrated (Kwan et al., 2015b)(Bhatnagar et al., 2016).

By demonstrating that extravasated particles can be mapped on a clinically relevant spatial scale, the system may in future be applied to an in vivo model to determine the spatial extent of co-delivered therapeutic agents of a similar size, thereby providing an indication of drug delivery.

\section{Conclusion}

This work demonstrates for the first time that it is possible to re-excite cavitation nuclei embedded within a viscoelastic matrix after they have been extravasated under ultrasound (for the purpose of ultrasound-mediated drug delivery applications), and once they are no longer present in flow. The location and extent of nuclei extravasation can be determined by Passive Acoustic Mapping of sources of broadband acoustic emissions associated with cavitation activity from these sub-micron extravasated nuclei. There was clear evidence of co-location of PAM signal with optical imaging of extravasated drug, with the average maximum distance of a PAM pixel from an extravasated particle being $0.4 \pm 0.2 \mathrm{~mm}$ for a dual array configuration used in conjunction with an adaptive beamformer. The spatial correlation between post-extravasation cavitation maps and extravasated drug depends significantly on the array aperture, and on the beamforming algorithms used. Whilst the dual linear array configuration demonstrates the best performance for this application, geometric constraints limit the applicability of this setup. Therefore, a single array configuration has also been evaluated with the aforementioned beamformers, and in this case, the RLPB algorithm demonstrates the best performance in terms of source localisation. However further work is required to ensure that adequate source localisation and time resolution can be achieved with a single linear array. Time resolution improvements may include the optimisation of the RLPB algorithm using commercially available purpose-built 
software and other data reduction strategies. For increased localisation performance in both the single and dual array configurations, image processing techniques such as attenuation and diffraction correction, and superresolution can be employed. Future work will include testing whether this technique can be used to fully map all extravasated particles (by steering the focus or increasing the concentration of cavitation nuclei), given that the present work has shown that nuclei can be reexcited once extravasated. In addition, this process will be applied in vivo which, if successful, may provide an indication of the spatial distribution of co-administered therapeutics in tumour tissue, and hence become a marker of treatment efficacy.

\section{Acknowledgements}

The authors would like to thank the members of the BUBBL group for their valuable advice and discussions regarding this work, in particular Dr Michael Gray and Dr Christophoros Mannaris. In addition, the authors thank the Institute of Biomedical Engineering Workshop Technicians, Jim Fisk and David Salisbury, for their support in the design and production of custom parts used in the experiments. Catherine Paverd is a Commonwealth Scholar funded by the UK government. Dr Erasmia Lyka and Dr Delphine Elbes are supported by the Engineering and Physical Sciences Research Council (EPSRC) under grant number EP/K020757/1. This research is further supported by the Oxford Centre for Drug Delivery Devices under the Engineering and Physical Research Council (EPSRC) programme grant $\mathrm{EP} / \mathrm{L} 024012 / 1$.

\section{References}

Arvanitis, C., Crake, C., McDannold, N., and Clement, G. (2016). Passive Acoustic Mapping with the Angular spectrum method. IEEE Trans. Med. Imaging, 36(4):983-993.

Arvanitis, C. D., Livingstone, M. S., Vykhodtseva, N., and McDannold, N. (2012). Controlled Ultrasound-Induced Blood-Brain Barrier Disruption Using Passive Acoustic Emissions Monitoring. PLoS One, 7(9).

Bazan-Peregrino, M., Arvanitis, C. D., Rifai, B., Seymour, L. W., and Coussios, C. C. (2012). Ultrasound-induced cavitation enhances the delivery and therapeutic efficacy of an oncolytic virus in an in vitro model. J. Control. Release, $157(2): 235-242$.

Bazan-Peregrino, M., Rifai, B., Carlisle, R. C., Choi, J., Arvanitis, C. D., Seymour, L. W., and Coussios, C. C. (2013). Cavitation-enhanced delivery of a replicating oncolytic adenovirus to tumors using focused ultrasound. J. Control. Release, 169(1-2):40-47. 
Bhatnagar, S., Kwan, J., Shah, A., Coussios, C., and Carlisle, R. (2016). Exploitation of sub-micron cavitation nuclei to enhance ultrasound-mediated transdermal transport and penetration of vaccines. Journal of Controlled Release.

Carlisle, R., Choi, J., Bazan-Peregrino, M., Laga, R., Subr, V., Kostka, L., Ulbrich, K., Coussios, C.-C., and Seymour, L. W. (2013). Enhanced Tumor Uptake and Penetration of Virotherapy Using Polymer Stealthing and Focused Ultrasound. JNCI: Journal of the National Cancer Institute, 105(22):1701-1710.

Choi, J. J., Carlisle, R. C., Coviello, C., Seymour, L., and Coussios, C.-C. (2014). Non-invasive and real-time passive acoustic mapping of ultrasound-mediated drug delivery. Phys. Med. Biol., 59(17):4861-77.

Collin, J. R. T. and Coussios, C. C. (2011). Quantitative observations of cavitation activity in a viscoelastic medium. J. Acoust. Soc. Am., 130(5):3289-3296.

Coviello, C., Kozick, R., Choi, J., Gyongy, M., Jensen, C., Smith, P. P., and Coussios, C.-C. (2014). Passive acoustic mapping utilizing optimal beamforming in ultrasound therapy monitoring. JASA.

Datta, S., Coussios, C. C., McAdory, L. E., Tan, J., Porter, T., De CourtenMyers, G., and Holland, C. K. (2006). Correlation of cavitation with ultrasound enhancement of thrombolysis. Ultrasound in Medicine and Biology, 32(8):12571267.

Dellian, M., Yuan, F., Trubetskoy, V. S., Torchilin, V. P., and Jain, R. K. (2000). Vascular permeability in a human tumour xenograft: molecular charge dependence. British Journal of Cancer, 82(9):1513-1518.

Dijkmans, P. A., Juffermans, L. J. M., Musters, R. J. P., Wamel, A. V., Cate, F. J., Gilst, W. V., Visser, C. A., Jong, N. D., and Kamp, O. (2004). Microbubbles and ultrasound : from diagnosis to therapy. Echocardiography, 5(November):245-256.

Feigenbaum, H., Stone, J. M., Don, A., and Nasser, W. K. (1970). Identification of Ultrasound Echoes from the Left Ventricle by Use of Intracardiac Injections of Indocyanine Green. Circulation, 41(4).

Gray, M. D., Lyka, E., and Coussios, C. C. (2018). Diffraction Effects and Compensation in Passive Acoustic Mapping. IEEE Trans. Ultrason. Ferroelectr. Freq. Control, 65(2):258-268.

Gyöngy, M., Arora, M., Noble, J. A., and Coussios, C. C. (2008). Use of Passive Arrays for Characterization and Mapping of Cavitation Activity during HIFU Exposure. In IEEE Int. Ultrason. Symp., pages 871-874.

Gyöngy, M. and Coussios, C. C. (2010). Passive cavitation mapping for localization and tracking of bubble dynamics. J. Acoust. Soc. Am., 128(4):EL175-EL180.

Haworth, K. J., Mast, T. D., Radhakrishnan, K., Burgess, M. T., Kopechek, J. A., Huang, S.-L., McPherson, D. D., and Holland, C. K. (2012). Passive imaging with pulsed ultrasound insonations. J. Acoust. Soc. Am., 132(1):544-553. 
Hobbs, S. K., Monsky, W. L., Yuan, F., Roberts, W. G., Torchilin, V. P., Jain, R. K., Robertsii, W. G., and Griffitht, L. (1998). Regulation of Transport Pathways in Tumor Vessels : Role of Tumor Type and Microenvironment. Proc. Natl. Acad. Sci. U. S. A., 95.

Hynynen, K., McDannold, N., Sheikov, N. A., Jolesz, F. A., and Vykhodtseva, N. (2005). Local and reversible blood-brain barrier disruption by noninvasive focused ultrasound at frequencies suitable for trans-skull sonications. NeuroImage, 24(1):12-20.

Hynynen, K., Pomeroy, O., Smith, D. N., Huber, P. E., McDannold, N. J., Kettenbach, J., Baum, J., Singer, S., and Jolesz, F. A. (2001). MR Imagingguided Focused Ultrasound Surgery of Fibroadenomas in the Breast: A Feasibility Study. Radiology, 219(1):176-185.

Jensen, C. R., Cleveland, R. O., and Coussios, C. C. (2013). Real-time temperature estimation and monitoring of HIFU ablation through a combined modeling and passive acoustic mapping approach. Phys. Med. Biol., 58(17):5833-5850.

Jiang, X., Zeng, W. J., Yasotharan, A., So, H. C., and Kirubarajan, T. (2014). Robust beamforming by linear programming. IEEE Trans. Signal Process., 62(7):1834-1849.

Keller, J. and Miksis, M. (1980). Bubble Oscillations of large Amplitude. JASA, 68(2):628-633.

Kennedy, J. E., Wu, F., Ter Haar, G. R., Gleeson, F. V., Phillips, R. R., Middleton, M. R., and Cranston, D. (2004). High-intensity focused ultrasound for the treatment of liver tumours. Ultrasonics, 42(1-9):931-935.

Kwan, J., Graham, S., Myers, R., Carlisle, R., Stride, E., and Coussios, C. (2015a). Ultrasound-induced inertial cavitation from gas-stabilizing nanoparticles. Phys. Rev. E, 023019(August):1-5.

Kwan, J., Myers, R., Coviello, C., Graham, S., Shah, A., Stride, E., Carlisle, R., and Coussios, C. (2015b). Ultrasound-Propelled Nanocups for Drug Delivery. Small, 11(39):5305-5314.

Lu, S., Hu, H., Yu, X., Long, J., Jing, B., Zong, Y., and Wan, M. (2018). Passive acoustic mapping of cavitation using eigenspace-based robust Capon beamformer in ultrasound therapy. Ultrason. Sonochem., 41(October 2017):670-679.

Lyka, E., Coviello, C. M., Paverd, C., Gray, M. D., and Coussios, C. C. (2018). Passive acoustic mapping using data-adaptive beamforming based on higher order statistics. IEEE Transactions on Medical Imaging, 37(12):2582-2592.

Mitragotri, S., Blankschtein, D., and Langer, R. (1995). Ultrasound-mediated transdermal protein delivery. Science, 269(5225):850-853.

Narayanan, J., Xiong, J.-Y., and Liu, X.-Y. (2006). Determination of agarose gel pore size: Absorbance measurements vis a vis other techniques. J. Phys. Conf. Ser., 28(1):83-86. 
Netti, P., Berk, D., Swartz, M., Grodzinsky, J., and Jain, R. K. (2000). Role of extracellular matrix assembly in interstitial transport in solid tumors. Cancer Research, 60:2497-2503.

O'Reilly, M. A. and Hynynen, K. (2013). A super-resolution ultrasound method for brain vascular mapping. Med. Phys., 40(11):110701.

Peer, D., Karp, J. M., Hong, S., Farokhzad, O. C., Margalit, R., and Langer, R. (2007). Nanocarriers as an emerging platform for cancer therapy. Nat. Nanotechnol., 2(12):751-760.

Ridler, T. (1978). Picture Thresholding Using an Iterative Slection Method. IEEE Trans. Syst. Man Cybern., 8(8):630-632.

Salgaonkar, V. A., Datta, S., Holland, C. K., and Mast, T. D. (2009). Passive cavitation imaging with ultrasound arrays. J. Acoust. Soc. Am., 126(6):30713083.

Sirsi, S. and Borden, M. (2010). Microbubble Compositions, Properties and Biomedical Applications. Bubble Sci Eng Technol., 1:3-17.

Uzgiris, E. E. (2008). Tumor microvasculature: endothelial leakiness and endothelial pore size distribution in a breast cancer model. Breast Cancer (Auckl)., 1:83-90.

Vaezy, S., Shi, X., Martin, R. W., Chi, E., Nelson, P. I., Bailey, M. R., and Crum, L. A. (2001). Real-time visualization of high-intensity focused ultrasound treatment using ultrasound imaging. Ultrasound Med Biol, 27(1):33-42.

Vlaisavljevich, E., Aydin, O., Yuksel Durmaz, Y., Lin, K.-W., Fowlkes, B., Xu, Z., and ElSayed, M. (2016). Effect of Droplet Compostition on Nanodroplet Mediated Histotripsy. Ultrasound in Medicine and Biology, 42(4). 